\title{
Tumor-draining lymph nodes are pivotal in PD-1/PD-L1 checkpoint therapy
}

\author{
Marieke F. Fransen, ${ }^{1}$ Mark Schoonderwoerd, ${ }^{2}$ Philipp Knopf, ${ }^{3}$ Marcel G.M. Camps, ${ }^{1}$ \\ Lukas J.A.C. Hawinkels, ${ }^{2}$ Manfred Kneilling, ${ }^{3,4}$ Thorbald van Hall, ${ }^{5}$ and Ferry Ossendorp ${ }^{1}$ \\ 'Department of Immunohematology and Blood Transfusion, Leiden University Medical Center (LUMC), Leiden, \\ Netherlands. ${ }^{2}$ Department of Gastroenterology and Hepatology, LUMC, Leiden, Netherlands. ${ }^{3}$ Department of Preclinical \\ Imaging and Radiopharmacy, Werner Siemens Imaging Center, Eberhard Karls University Tuebingen, Tuebingen, Germany. \\ ${ }^{4}$ Department of Dermatology, Eberhard Karls University Tuebingen, Tuebingen, Germany. ${ }^{5}$ Department of Medical \\ Oncology, LUMC, Leiden, Netherlands.
}

PD-1/PD-L1 checkpoint therapy for cancer is commonly considered to act by reactivating $T$ cells in the tumor microenvironment. Here, we present data from 2 mouse tumor models demonstrating an essential involvement of tumor-draining lymph nodes in PD-1 and PD-L1 therapeutic efficacy. Immune activation induced by checkpoint treatment was predominantly observed in the tumordraining, but not nondraining, lymph nodes and was reflected in local accumulation of CD8 ${ }^{+}$T cells. Surgical resection of these lymph nodes, but not contralateral lymph nodes, abolished therapyinduced tumor regressions and was associated with decreased immune infiltrate in the tumor microenvironment. Moreover, inhibitor FTY720, which locks lymphocytes in lymph organs, also abrogated checkpoint therapy, suggesting that the tumor-draining lymph nodes function as sites of $T$ cell invigoration required for checkpoint blockade therapy. Now that PD-1/PD-L1 checkpoint treatment is applied in earlier clinical stages of cancer, our preclinical data advocate for enrolling patients with their tumor-draining lymph nodes still in place, to optimally engage the antitumor immune response and thereby enhance clinical benefit.

Authorship note: TVH and FO are co-senior authors.

Conflict of interest: The authors have declared that no conflict of interest exists.

License: Copyright 2018, American Society for Clinical Investigation.

Submitted: August 28, 2018 Accepted: October 29, 2018 Published: December 6, 2018

\section{Reference information:} JCI Insight. 2018;3(23):e124507. https://doi.org/10.1172/jci. insight.124507.

\section{Introduction}

Blocking antibodies against PD-1 and PD-L1, named checkpoint molecules, shows exceptional clinical responses in cancer patients and has already become a standard-of-care treatment in a still-increasing number of cancer types (1). Generally, PD-1/PD-L1 checkpoint blockers are thought to invigorate T cells within the tumor microenvironment (TME), where PD-L1 is expressed on tumor cells and infiltrating myeloid cells. Indeed, therapeutic responses generally correlate with high T cell infiltrate, PD-L1 expression, and tumor mutational load (2). More recent studies have pointed to systemic factors, such as frequencies of myeloid cells, lymphocytes, and eosinophils in peripheral blood (3-5), and we and others have shown that PD-L1 expression on tumor cells is not a prerequisite for successful checkpoint therapy (6-9). However, the exact mode of action in vivo is still poorly understood. Thus far, studies on the role of tumor-draining lymph nodes (TDLNs) for checkpoint therapy are limited. TDLNs are the first sites of metastasis and are therefore often resected when invaded by tumor cells. Lymph nodes (LNs) play important roles in the generation and regulation of immune responses to pathogens and autoantigens (10). TDLNs have been described to contain both tumor effector as well as suppressor immune components (11), and it has been shown that tumor antigens drain primarily to TDLNs, more specifically via transportation by CD103+ DCs, which leads to priming of T cells (12-14).

We here investigate the role of TDLNs in checkpoint therapy of solid tumors in preclinical mouse models, by analyzing effects of checkpoint therapy on TDLN immune content and surgically resecting TDLNs before checkpoint therapy.

\section{Results}

We set out to evaluate the role of TDLNs in PD1/PD-L1 therapy by analyzing immune cell composition of TDLNs and nondraining lymph nodes (NDLNs). MC38 colon carcinoma cells were inoculated subcutaneously in the flank of syngeneic mice, and TDLNs (inguinal and axillary LNs) were isolated for in vitro analyses, 
after verification of drainage with fluorescent imaging (data not shown). LNs at the opposite flank of the mice served as internal, nondraining controls. We observed that $\mathrm{CD} 11 \mathrm{~b}^{+}$myeloid cells in the TDLNs of untreated tumor-bearing mice expressed higher PD-L1 levels as compared with levels in NDLNs, suggesting that active immune suppression is ongoing (Figure 1A). A strong increase in cellularity was observed in TDLNs 3 days after the start of PD-1-blocking treatment (Figure 1B). This swelling did not cause gross irregularities in LN architecture (Supplemental Figure 1; supplemental material available online with this article; https://doi.org/10.1172/ jci.insight.124507DS1). PD-1 treatment resulted in an increase of the total number of CD8 $8^{+} \mathrm{T}$ cells in TDLNs. Activated $\mathrm{CD}^{+} \mathrm{T}$ cells were more abundant in TDLNs compared with NDLNs as indicated by the proliferation marker Ki67 and transcription factor T-bet (Figure 1C). In contrast, hardly any T cell activation was found in NDLNs or LNs from tumor-free, PD-1-treated mice, indicating that vigorous $\mathrm{T}$ cell immune activation after $\mathrm{PD}-1-$ blocking $\mathrm{Ab}$ treatment within TDLNs depends on the presence of tumors.

This suggested that PD-1 immunotherapy could activate T cells in the TDLNs, which may contribute to the antitumor response. By surgically resecting inguinal and axillary TDLNs just before the start of PD-1 treatment, we evaluated the actual contribution of TDLNs in therapeutic efficacy. In the absence of TDLNs, the treatment efficacy was strongly diminished (Figure 2, A and B), compared with mock surgery controls. We previously demonstrated that local administration, close to the tumor site, of immunomodulatory Abs, such as agonistic anti-CD40 and blocking anti-CTLA-4, decreased toxicity but sustained the treatment effect $(15,16)$. $\mathrm{PD}$-1-blocking $\mathrm{Ab}$ was also operational in this setting (Figure $2 \mathrm{C}$ ), suggesting that neighboring communication between tumor and TDLN is critical. We then reasoned that draining of tumor-derived antigens to the TDLNs might be involved in this system and resected the TDLNs even before tumor inoculation. In this setting, PD-1 efficacy was completely abrogated, whereas resection after tumor settlement, as applied thus far, still showed residual tumor control (Figure 2C). To control for a nonspecific effect of disrupting the lymphoid system, we resected the NDLN at the opposite flank, which did not influence the therapeutic efficacy (Figure 2D and Supplemental Figure 2). Finally, we applied this treatment setting with PD-L1-blocking Ab in another colon tumor model on a distinct genetic background, CT26 in BALB/c mice. In this independent model, PD-L1 blockade displayed an identical dependency on the presence of the TDLNs (Figure 2E).

These results suggested that $\mathrm{T}$ cell trafficking was required for therapeutic efficacy; we therefore treated tumor-bearing mice with PD-1 therapy in the presence of the S1P receptor inhibitor FTY720, which locks T cells in lymphoid organs. Efficacy of FTY720 administration was shown by decrease in T cell content of peripheral blood (Figure 3A). Importantly, FTY720 mitigated the therapeutic efficacy of PD-1 treatment (Figure 3B), suggesting that therapeutic efficacy is not solely based on reactivation of $\mathrm{T}$ cells within the TME but that influx of $\mathrm{T}$ cells from elsewhere dominantly contributes to this therapy.

Next we analyzed the TME of tumor-bearing mice treated with PD-1-blocking Ab with or without TDLN resection. We found a strong decrease of $\mathrm{CD} 45^{+}$immune infiltrate in the TME of TDLN-resected mice, pointing at a role for TDLNs in trafficking of immune cells to the tumor (Figure 3C). Additionally, significantly more $\mathrm{CD}^{+} \mathrm{T}$ cells were found in the TME of TDLN-proficient versus TDLN-deficient mice after PD-1 treatment. Proliferation marker Ki67 did not differ between CD8 ${ }^{+} \mathrm{T}$ cells of these groups, suggesting that influx of cells, rather than enhanced proliferation of T cells within the TME, caused this difference. Together, our data indicated that PD-1 blockade reinvigorates CD8 ${ }^{+} \mathrm{T}$ cells in the TDLNs, resulting in an influx of these effector cells to the TME.

\section{Discussion}

In conclusion, we show that TDLNs are key regulators in the antitumor immune response and control the magnitude of therapeutic efficacy of PD-1- and PD-L1-blocking Ab treatment in mouse models. This is in agreement with a previous study analyzing the role of VEGF-C. Increased VEGF-C in the tumor caused enhanced lymph drainage, which was associated with increased antitumor immune response and stronger effect of immunotherapy (17). These data strongly support our findings, suggesting a potential reservoir of activating tumor-specific immune cells within TDLNs.

Harnessing this empowering effect of TDLNs might benefit the clinical outcome of checkpoint blockade therapy and might ideally be tested in early-stage cancer patients where the tumor and TDLNs are still in place. Applying therapy before resection surgery, often termed neoadjuvant treatment, is already performed for chemotherapy and radiotherapy to reduce tumor size before surgery and to pre-evaluate therapy response. For immunotherapy, this strategy is only starting to be explored (18-21). The potential of TDLNs is demonstrated in the clinical study of Koster et al in which stage I and II melanoma patients were treated with immune-stim- 
PD-L1 expression

A myeloid cells

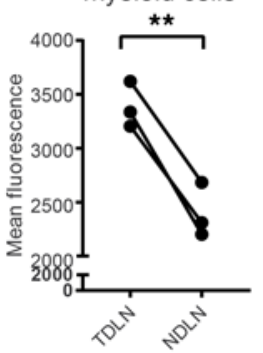

$\mathbf{C}$

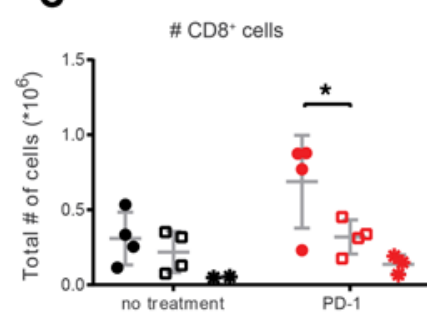

B Cell count in LN day 3

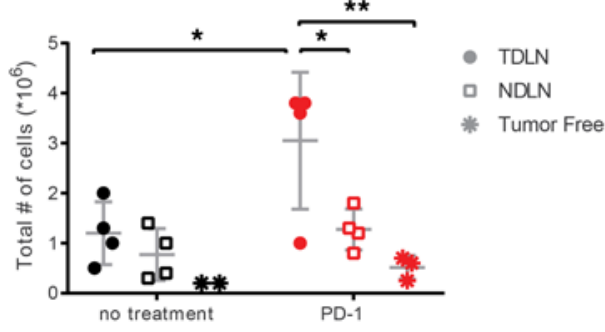

Figure 1. Immune activation takes place in tumor-draining, but not in nondraining, lymph nodes. (A) TDLNs (inguinal and axillary) and NDLNs (opposite flank) were isolated when subcutaneous MC38 tumors reached an average size of $100 \mathrm{~mm}^{3}$ and were analyzed with flow cytometry. Mean fluorescence expression of PD-L1 on myeloid cells (CD19-CD11b+) is shown. Statistical difference was calculated with a paired 2-tailed $t$ test. (B and C) Three days after PD-1 Ab treatment, lymph nodes were analyzed for (B) immune cell counts and numbers of (C, left) $C D 8^{+} T$ cells, (C, middle) proliferating CD8 $8^{+}$cells, and (C, right) T-bet $^{+} \mathrm{CD}^{+} \mathrm{T}$ cells. Statistical differences were analyzed with 2-way ANOVA. All data represent mean \pm SEM from 1 experiment ( $n=4$ per group) out of 3 independent experiments with similar outcome $\left({ }^{*} P<0.05,{ }^{* *} P<0.01\right)$.

ulating TLR9 agonist CpG in the scar of tumor resection, before TDLN resection. Patients displayed stronger tumor-specific immune responses and longer recurrence-free survival than placebo-injected patients (22).

Recently, in a preclinical mouse study, treatments with PD-1 combined with other agents in an adjuvant (the tumor and TDLN are resected) or neoadjuvant setting (the tumor and TDLN are in place) were compared and showed improved outcomes for neoadjuvant treatment (18). The enhanced tumor-specific $\mathrm{T}$ cell response and decrease of metastatic recurrence was attributed to the activation of immune cells within the TME. In light of our findings, it is conceivable that the TDLNs, which were also surgically resected in the adjuvant group, were responsible for this, but this was not taken into account.

Sentinel LN resection is still common clinical practice in many forms of cancer to determine disease staging and consecutive therapy options, and when metastases are detectable in the sentinel LN, resection of several, if not all, draining LNs is often performed. There is ongoing debate on the value of this strategy, with regard to predictive value, metastasis risk, and consequences for treatment options (23). However, the immunological implications of $\mathrm{LN}$ resection are not evaluated here. We now show that ignoring a potential positive role of TDLNs in immunotherapy for cancer should be studied with care in clinical settings.

\section{Methods}

Mice and cell lines. C57BL/6 mice and BALB/c mice were purchased from Charles River and housed under specified pathogen-free conditions in animal facilities of Leiden University Medical Center. MC38 and CT26 cells were cultured in IMDM (Lonza) containing 8\% FCS (Greiner), $100 \mathrm{IU} / \mathrm{ml}$ penicillin/streptomycin (Gibco), $2 \mu \mathrm{M}$ glutamin (Gibco), and $25 \mu \mathrm{M}$ 2-mercaptoethanol. Cell lines were mycoplasma tested and MAP tested before the start of experiments.

Tumor inoculation. Tumors were inoculated by subcutaneous injection in the right flank of 250,000 MC38 cells or 100,000 CT26 cells in $100 \mu 1$ PBS. Tumor outgrowth was measured in 3 dimensions, until mice had to be sacrificed due to tumor burden, according to local ethical guidelines.

Treatments. Tumor-bearing mice were treated on days 7 and 10 after tumor inoculation by intraperitoneal injection of $200 \mu \mathrm{g}$ PD-1-blocking Ab (clone RMP1-14 from Bio X Cell or BioLegend) or PD-L1blocking Ab (clone 10F.9G2, Bio X Cell), or peritumoral subcutaneous injection of $50 \mu \mathrm{g}$ PD-1-blocking $\mathrm{Ab}$ (clone RMP1-14, Bio X Cell). Tumor size was checked 2 or 3 times a week and measured in 3 dimen- 


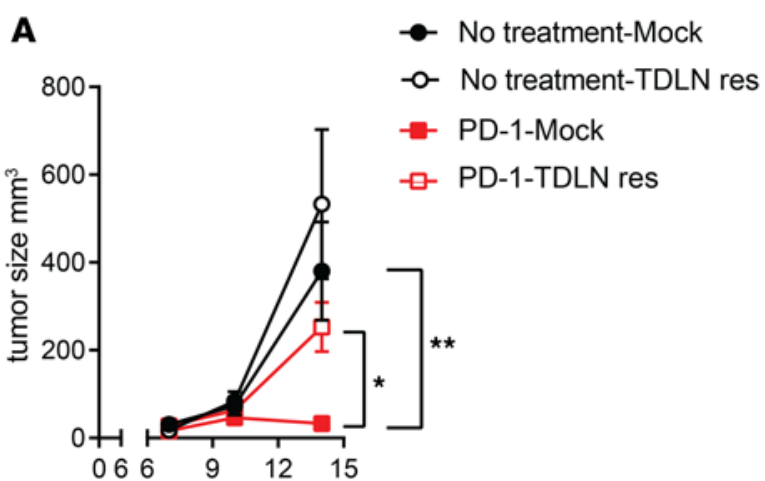

Days after tumor inoculation

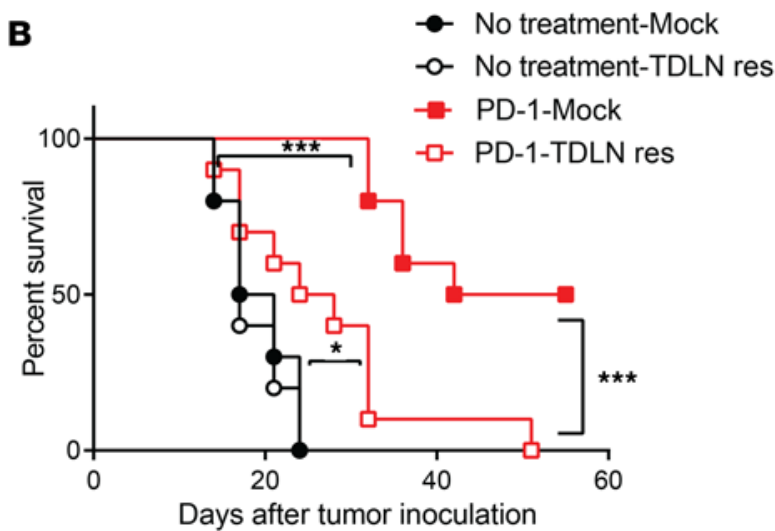

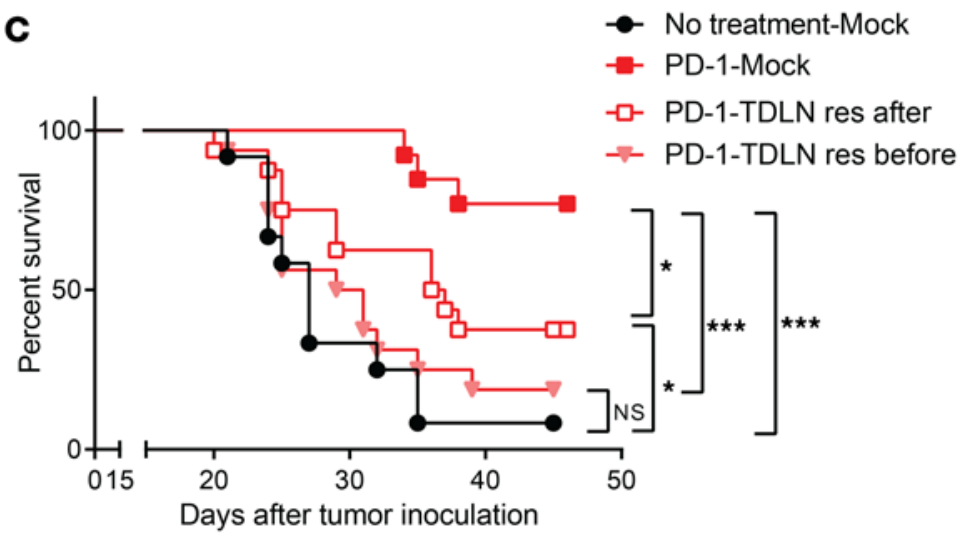

D

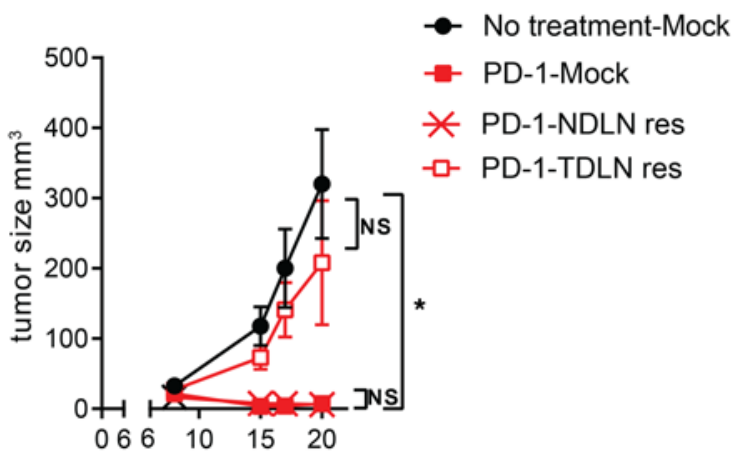

Days after tumor inoculation

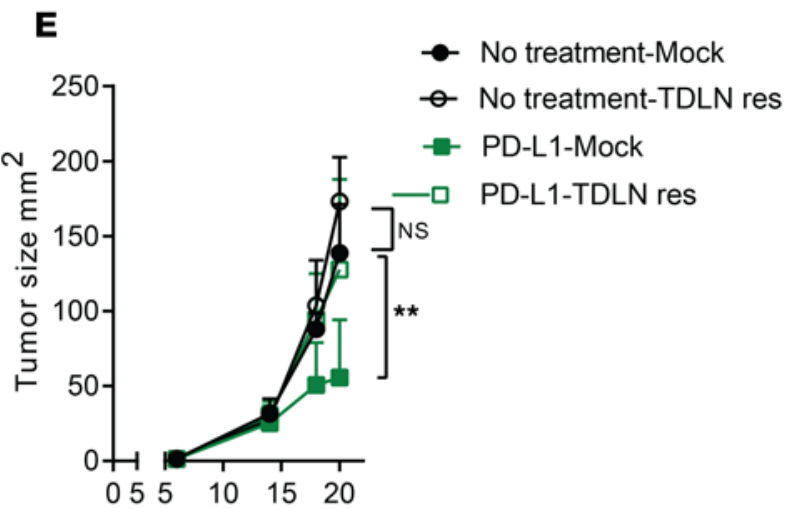

Days after tumor inoculation

Figure 2. Essential role of TDLNs in checkpoint therapy. Mice bearing subcutaneous tumors in the right flank were treated with checkpoint blocker therapy immediately following lymph node resection surgery or mock surgery. (A-D) C57BL/6 mice bearing MC38 tumors were treated with PD-1-blocking Ab immediately following lymph node resection surgery. (A) Average outgrowth of mice treated with PD-1 Ab systemically $(2 \times 100 \pi \mathrm{g}$ i.p.). Average \pm SEM are depicted. Statistical analysis of average tumor outgrowth difference on day 14 was calculated by 1-way ANOVA, 10 mice per group. Res: resection. (B) Survival of mice treated with PD-1 Ab systemically $(2 \times 100 \pi$ g i.p.). Statistical difference was analyzed with log-rank test, 10 mice per group. (C) Survival of mice treated with PD-1 Ab systemically ( $2 \times 100 \pi \mathrm{g}$ i.p.). TDLNs were resected 7 days after ("res after") or 1 day before ("res before") tumor inoculation. Pooled data of 2 independent experiments, with 16 mice per group. Statistical difference was analyzed with log-rank test. (D) Average tumor outgrowth of mice treated with local injection of low-dose PD-1 Ab ( $1 \times 50 \pi$ s.c.), with TDLNs or NDLNs resected before PD- 1 treatment, 8 mice per group. Average \pm SEM are depicted. Statistical difference in average tumor size on day 20 was calculated by 1-way ANOVA. (E) BALB/c mice bearing subcutaneous CT26 tumors in the right flank were treated with PD-L1 Ab systemically. Average tumor outgrowth \pm SEM are depicted. One representative experiment is shown from 2 performed, with 8 mice per group. Statistical analysis of average tumor size on day 20 was calculated with 1 -way ANOVA $\left({ }^{*} P<0.05,{ }^{* *} P<0.01\right.$, ${ }^{* * *} P<0.005$, NS: nonsignificant). 

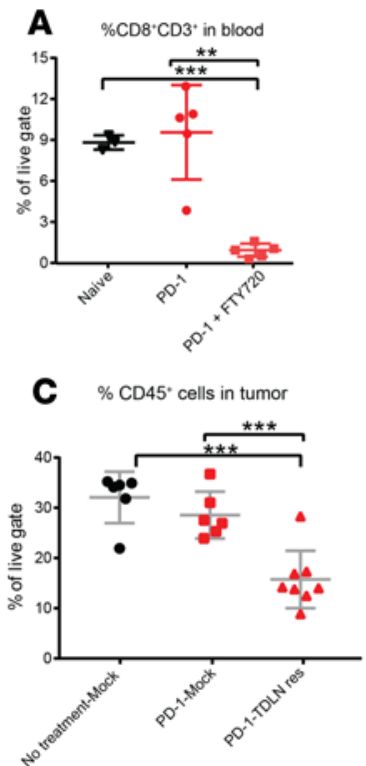

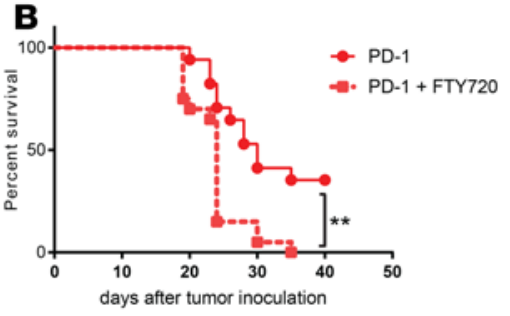

D $\% \mathrm{CD} 8^{*}$ cells of $\mathrm{CD} 45^{*}$ in tumor

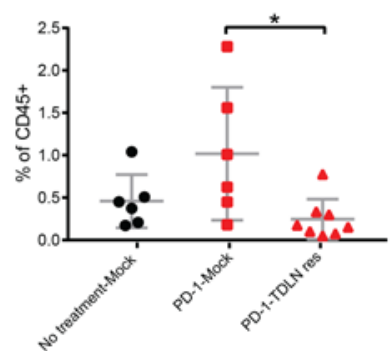

E $\% \mathrm{Ki}^{\circ} 7^{*}$ of $\mathrm{CD} 88^{*}$ cells in tumor

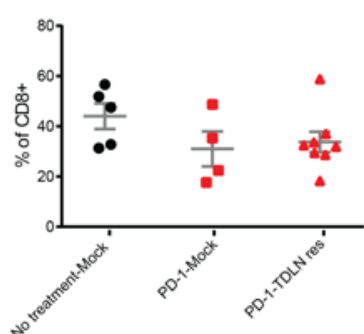

Figure 3. Increase of intratumoral CD8 ${ }^{+} \mathrm{T}$ cell numbers after PD-1 treatment is abrogated in the absence of TDLNs. (A) $\mathrm{CD3}^{+} \mathrm{CD}^{+}$content in peripheral blood of mice treated with FTY720. Mean \pm SEM are depicted. Statistical differences were calculated with 1-way ANOVA. (B) Survival of mice treated with PD-1-blocking Ab with or without FTY720. Pooled data of 2 comparable experiments with 16 mice per group are shown. Statistical analysis was done by log-rank test. (C-E) Analysis of subcutaneous MC38 tumors at day 13 after PD-1 treatment, with or without TDLN resection immediately before PD-1 treatment. (C) Percentage of CD45+ cells out of live gate. (D) Percentage of CD8+ cells out of CD45+ gate. (E) Percentage of $\mathrm{Ki}^{+}$cells out of CD8 ${ }^{+}$cells. Statistical analysis was performed with 1-way ANOVA, 5-8 mice per group $\left({ }^{*} P<0.05,{ }^{* *} P<0.01,{ }^{* *} P<0.005\right) ; 1$ representative experiment is shown out of 2 performed.

sions. FTY720 treatment was given intraperitoneally, $25 \mu \mathrm{g}$ in saline, on days 6, 8, 10, 13, 16, and 20. Retention of lymphocytes in lymphoid organs was confirmed on day 16 (before subsequent injection) in peripheral blood by flow cytometry (Supplemental Figure 2B).

Surgical resection. Mice received buprenorphine (Temgesic) painkiller preoperatively ( $3 \mu \mathrm{g}$ per mouse, subcutaneously), after which they were anesthetized with isoflurane inhalation and the right flank was subsequently shaved. Small incisions were made in the groin area (for inguinal) and armpit (for axillary). Inguinal or axillary LNs were located using blunt forceps and resected using sharp forceps. Control animals were mock resected; similar incisions were made but LNs were left in place. Incisions were sutured using Novosyn Quick suture 6/0 (B. Braun), and mice were placed back in their cage.

Flow cytometry. Cell surface staining was performed using the following Abs: CD8 $\alpha$ (clone 53-6.7), CD3 $\varepsilon$ (clone 145-2c11), CD11b (clone M1/70), CD45.2 (clone 104), and PD-L1 (clone MIH5). Dead cells were excluded based on 7-aminoactinomycin D (Invitrogen). After surface staining, cells were fixed with Foxp3 staining kit (eBioscience), and cells were stained with Ki67 (clone B56) and T-bet (clone eBio4B10). Examples of gating strategies are depicted in Supplemental Figure 3. Samples were analyzed with LSR II cytometer (BD Biosciences) using FACSDiva software (BD Biosciences) and FlowJo software (Tree Star Inc). For flow cytometry analyses of TME, mice were perfused with PBS/EDTA $(2 \mu \mathrm{M})$ to exclude blood content. Tumors were isolated, minced with scalpels, and incubated with $2.5 \mathrm{mg} / \mathrm{ml}$ Liberase TL (Roche) for 20 minutes at $37^{\circ} \mathrm{C}$, and single-cell suspensions were made using $70-\mu \mathrm{m}$ cell strainers (BD Biosciences).

Histology. MC38-bearing mice were left untreated or received $200 \mu \mathrm{g}$ of PD-1 Ab intraperitoneally on day 7 after tumor inoculation. Inguinal TDLNs and NDLNs were isolated 3 days later and fixed in formalin dehydrated in series of increasing amounts of ethanol. Tissue was embedded in paraffin, sequentially sectioned, and stained with an H\&E. Photos were taken using an Olympus DX51 light microscope and Olympus cellSens software. From each organ, the largest section was chosen.

Data availability. All data are available from the corresponding authors upon reasonable request.

Statistics. GraphPad Prism 7 software was used for all statistical analyses. The means of groups were compared using $t$ test or ANOVA (depending on how many groups, and survival differences in Kaplan-Meier curves were analyzed by log-rank test; all tests were 2-tailed. Differences were considered statistically 
significant at $P$ value less than $0.05\left({ }^{*} P<0.05,{ }^{* *} P<0.01,{ }^{* *} P<0.005\right)$. For flow cytometry analysis of TME, Grubbs' test for outliers was performed on tumor size within their groups, and significant outliers were removed from all analyses.

Study approval. All animal experimentations were approved by and conducted according to guidelines of the Netherlands Association of Animal Experimentation Committees (Central Animal Testing Commission, the Hague, the Netherlands).

\section{Author contributions}

MFF, MS, PK, and MGMC performed experiments and acquired and analyzed data. MFF, MS, LJACH, MK, TVH, and FO interpreted data. MFF, TVH, and FO conceived and designed the study. MFF, MS, $\mathrm{TVH}$, and FO wrote the manuscript. All authors contributed to reviewing and revising the manuscript.

\section{Acknowledgments}

This work was supported by Dutch Cancer Society UL 2014-6828 (to MFF and MGMC) and Stichting Fonds Oncologie Holland (to MS and LJACH).

Address corresponcence to: Marieke F. Fransen, LUMC, Department of Immunohematology and Bloodtransfusion, Postzone E3Q, Albinusdreef 2, 2333 ZA Leiden, Netherlands. Phone: 31.71.5266832; Email: M.F.Herbert-Fransen@1umc.nl.

1. Topalian SL. Targeting immune checkpoints in cancer therapy. JAMA. 2017;318(17):1647-1648.

2. Binnewies M, et al. Understanding the tumor immune microenvironment (TIME) for effective therapy. Nat Med. 2018;24(5):541-550.

3. Spitzer MH, et al. Systemic immunity is required for effective cancer immunotherapy. Cell. 2017;168(3):487-502.e15.

4. Krieg C, et al. High-dimensional single-cell analysis predicts response to anti-PD-1 immunotherapy. Nat Med. 2018;24(2):144-153.

5. Weide B, et al. Baseline biomarkers for outcome of melanoma patients treated with pembrolizumab. Clin Cancer Res. 2016;22(22):5487-5496.

6. Lau J, et al. Tumour and host cell PD-L1 is required to mediate suppression of anti-tumour immunity in mice. Nat Commun. $2017 ; 8: 14572$.

7. Kleinovink JW, Marijt KA, Schoonderwoerd MJA, van Hall T, Ossendorp F, Fransen MF. PD-L1 expression on malignant cells is no prerequisite for checkpoint therapy. Oncoimmunology. 2017;6(4):e1294299.

8. Juneja VR, et al. PD-L1 on tumor cells is sufficient for immune evasion in immunogenic tumors and inhibits CD8 T cell cytotoxicity. J Exp Med. 2017;214(4):895-904.

9. Noguchi T, et al. Temporally distinct PD-L1 expression by tumor and host cells contributes to immune escape. Cancer Immunol Res. 2017;5(2):106-117.

10. Gasteiger G, Ataide M, Kastenmüller W. Lymph node - an organ for T-cell activation and pathogen defense. Immunol Rev. 2016;271(1):200-220.

11. Fransen MF, Arens R, Melief CJ. Local targets for immune therapy to cancer: tumor draining lymph nodes and tumor microenvironment. Int J Cancer. 2013;132(9):1971-1976.

12. Marzo AL, et al. Tumor antigens are constitutively presented in the draining lymph nodes. J Immunol. 1999;162(10):5838-5845

13. Hargadon KM, Brinkman CC, Sheasley-O'neill SL, Nichols LA, Bullock TN, Engelhard VH. Incomplete differentiation of antigen-specific CD8 T cells in tumor-draining lymph nodes. J Immunol. 2006;177(9):6081-6090.

14. Salmon H, et al. Expansion and activation of CD103(+) dendritic cell progenitors at the tumor site enhances tumor responses to therapeutic PD-L1 and BRAF inhibition. Immunity. 2016;44(4):924-938.

15. Fransen MF, van der Sluis TC, Ossendorp F, Arens R, Melief CJ. Controlled local delivery of CTLA-4 blocking antibody induces CD8+ T-cell-dependent tumor eradication and decreases risk of toxic side effects. Clin Cancer Res. 2013;19(19):5381-5389.

16. Fransen MF, Sluijter M, Morreau H, Arens R, Melief CJ. Local activation of CD8 T cells and systemic tumor eradication without toxicity via slow release and local delivery of agonistic CD40 antibody. Clin Cancer Res. 2011;17(8):2270-2280.

17. Fankhauser $\mathrm{M}$, et al. Tumor lymphangiogenesis promotes $\mathrm{T}$ cell infiltration and potentiates immunotherapy in melanoma. $S c i$ Transl Med. 2017;9(407):eaal4712.

18. Liu J, et al. Improved efficacy of neoadjuvant compared to adjuvant immunotherapy to eradicate metastatic disease. Cancer Discov. 2016;6(12):1382-1399.

19. Keung EZ, Ukponmwan EU, Cogdill AP, Wargo JA. The rationale and emerging use of neoadjuvant immune checkpoint blockade for solid malignancies. Ann Surg Oncol. 2018;25(7):1814-1827.

20. Amaria RN, et al. Neoadjuvant plus adjuvant dabrafenib and trametinib versus standard of care in patients with high-risk, surgically resectable melanoma: a single-centre, open-label, randomised, phase 2 trial. Lancet Oncol. 2018;19(2):181-193.

21. Forde PM, et al. Neoadjuvant PD-1 blockade in resectable lung cancer. N Engl J Med. 2018;378(21):1976-1986.

22. Koster BD, et al. Local adjuvant treatment with low-dose CpG-B offers durable protection against disease recurrence in clinical stage I-II melanoma: data from two randomized phase II trials. Clin Cancer Res. 2017;23(19):5679-5686.

23. Morton DL, et al. Final trial report of sentinel-node biopsy versus nodal observation in melanoma. N Engl J Med. 2014;370(7):599-609. 